\title{
Nodal Melanoma Metastasis under Infliximab Therapy in a Patient with Nevoid Melanoma First Misdiagnosed as Benign Nevus: A Potentially Dangerous Diagnostic Pitfall in the Era of Biologic Therapies
}

\author{
Gilles Safa ${ }^{a}$ Sophie Fromentoux ${ }^{a} \quad$ Laure Darrieux $^{a}$ \\ Jean-Anastase Hogenhuis ${ }^{b}$ Laurent Tisseau ${ }^{b}$ \\ ${ }^{a}$ Department of Dermatology, Centre Hospitalier de Saint-Brieuc, Saint-Brieuc, and \\ ${ }^{\mathrm{b}}$ Armor Pathology Laboratory, Plérin, France
}

\section{Key Words}

Nevoid melanoma $\cdot$ Metastasis $\cdot$ Infliximab $\cdot$ Tumor necrosis factor- $\alpha \cdot$ Diagnostic pitfalls

\begin{abstract}
We report the case of a 53-year-old Caucasian woman who developed nodal melanoma metastasis under infliximab therapy 2 years after the removal of a nevoid melanoma, which was initially misdiagnosed as a benign compound nevus. This case illustrates the potential link between tumor necrosis factor (TNF)- $\alpha$ inhibition and the reactivation of latent melanoma. Furthermore, this case highlights the need for a complete skin examination before using anti-TNF- $\alpha$ therapy to rule out atypical malignant lesions or melanomas that can easily be missed because of presentations such as nevoid melanoma.
\end{abstract}


Safa et al.: Nodal Melanoma Metastasis under Infliximab Therapy in a Patient with Nevoid Melanoma First Misdiagnosed as Benign Nevus: A Potentially Dangerous Diagnostic Pitfall in the Era of Biologic Therapies

\section{Introduction}

Although the risk of malignancy with tumor necrosis factor (TNF)- $\alpha$ inhibitors is still unclear, the majority of studies examining this carcinogenic risk suggest that TNF- $\alpha$ inhibitors may slightly increase the risk of cancer, including nonmelanoma skin cancer and hematologic malignancies [1]. Herein, we report the case of a patient who developed nodal melanoma metastasis under infliximab therapy 2 years after the removal of a nevoid melanoma that was first misdiagnosed as a benign compound nevus.

\section{Case Presentation}

A 53-year-old Caucasian woman presented with a 40-year history of severe plaque psoriasis. The patient had recalcitrant psoriasis that was unresponsive to multiple conventional therapies such as methotrexate and acitretin. The patient also had a 20 -year history $(>800$ treatments) of psoralen plus ultraviolet A (PUVA) therapy. Her past medical history was notable for a benign compound nevus on the left anterior thigh, which was completely excised in January 2011. In January 2012, the patient was screened (including physical examination, baseline laboratory studies, and screening for latent tuberculosis and chronic viral infections) and began etanercept therapy ( $50 \mathrm{mg}$ subcutaneously twice weekly). After 3 months, the patient was responding insufficiently to etanercept and was switched to infliximab (5 mg/kg at weeks 0,2 and 6, and then every 8 weeks thereafter). This treatment resulted in the complete disappearance of her psoriasis. Twelve months after beginning the infliximab treatment, the patient developed palpable lymphadenopathy in the left inguinal region. Given the suspect nature of the palpable mass, the patient underwent an inguinal lymph node biopsy. The histology of the biopsy specimen showed a metastatic melanoma. Positron emission and computed tomography scans were negative for distant metastatic disease. Infliximab was discontinued and a complete inguinal lymph node dissection was performed, which revealed no additional nodal involvement. A full skin evaluation did not reveal any suspicious lesions, but $4 \mathrm{~cm}$ below the metastatic lymph node, the scar from the previously removed nevus could be seen (fig. 1). The histopathologic slides of the excised nevus were reviewed along with immunohistochemical studies, which led to the definite diagnosis of nevoid melanoma (Breslow thickness $1.44 \mathrm{~mm}$; fig. 2). A BRAF V600E mutation was detected and the patient was enrolled in a clinical trial of combined adjuvant therapy with dabrafenib and trametinib.

\section{Discussion}

Recent clinical studies and case reports have suggested a possible link between antiTNF- $\alpha$ therapy and the occurrence of primary cutaneous melanoma or the reactivation of latent metastatic melanoma [2-4]. TNF- $\alpha$ is a cytokine of the innate immune system critical in the surveillance of malignancies and infections [4]. Experimental studies indicate that TNF- $\alpha$ plays an important role in the growth regulation of melanomas [5]. However, a causal relationship between TNF- $\alpha$ inhibitors and the development or the reactivation of melanoma remains to be established. In addition, the presence of other predisposing factors, such as the use of different immunosuppressive drugs, previous chronic sun exposure, and positive family history of melanoma, make the establishment of a causal link difficult [3]. It should be noted that our patient had been previously treated with multiple drug therapies including 
PUVA therapy and methotrexate. In a 25-year follow-up study of 1,380 patients with psoriasis who were first treated with PUVA, about 15 years after the first PUVA treatment, the occurrence of cutaneous melanomas was five times higher, especially among patients who received 250 treatments or more [6]. In an Australian cohort of patients with rheumatoid arthritis exposed to methotrexate, it was found that, compared to the general population, they had a 3-fold increased risk of melanoma. However, in Australia, the general population's risk of melanoma is also higher than in any other parts of the world because of the increased exposure to UV radiation [7]. Although the patient's history of long-term PUVA therapy may have been a major contributing factor to primary melanoma, the immunosuppressive effects of infliximab may also have played a role in the development of metastatic lymphadenopathy. In our case, although a clear causal relationship between TNF- $\alpha$ inhibitors and the reactivation of melanoma cannot be established, there is a temporal relationship between the initiation of infliximab therapy and the development of the lymph node positivity. The potential link between TNF- $\alpha$ inhibition and the reactivation of latent melanoma illustrated in this case suggests that TNF- $\alpha$ plays an important role in the immunosurveillance of melanoma micrometastases.

Nevoid melanoma refers to a rare and diagnostically deceptive histologic variant of melanoma with features that resemble an ordinary nevus. Therefore, this diagnosis can easily be missed [8]. Nevoid melanoma is a diagnostic challenge in dermatopathology and it is most often recognized either when it recurs locally or presents with a metastatic disease [9]. Such a misdiagnosis can have serious implications for patients treated with anti-TNF- $\alpha$ therapy and for the treating dermatologist and pathologist, who may be accused of medical malpractice. Histologically, nevoid melanoma has the low-power architecture of a banal compound or dermal nevus. At high power, histologic features favoring melanoma include mild atypia, slight increase in nucleolar prominence, slight impairment of maturation, and the usually inevitable presence of multiple mitotic figures in the dermal component. Immunohistochemical staining can be a useful diagnostic tool showing a high Ki-67 labeling index in the deeper component of the neoplasm [8-10].

\section{Conclusion}

This case emphasizes the need for a complete skin examination before using anti-TNF- $\alpha$ therapy. Furthermore, we recommend reviewing each previously excised cutaneous tumor of all patients who are eligible for biologic therapies, particularly in case of cutaneous melanocytic lesions, to rule out atypical malignant lesions or melanomas that can easily be missed because of presentations such as nevoid melanoma.

\section{Disclosure Statement}

The authors declare no conflict of interest.

\section{References}

1 Patel RV, Clark LN, Lebwohl M, Weinberg JM: Treatments for psoriasis and the risk of malignancy. J Am Acad Dermatol 2009;60:1001-1017. 
Safa et al.: Nodal Melanoma Metastasis under Infliximab Therapy in a Patient with Nevoid Melanoma First Misdiagnosed as Benign Nevus: A Potentially Dangerous Diagnostic Pitfall in the Era of Biologic Therapies

-2 Raaschou P, Simard JF, Holmqvist M, Askling J, ARTIS Study Group: Rheumatoid arthritis, anti-tumour necrosis factor therapy, and risk of malignant melanoma: nationwide population based prospective cohort study from Sweden. BMJ 2013;346:f1939.

-3 Mann J, Thomson P, Stevens H, Palamaras I: Malignant melanoma and tumor necrosis factor- $\alpha$ inhibitors: a case report and review of the literature. Int J Dermatol 2013;52:471-474.

-4 Fulchiero GJ Jr, Salvaggio H, Drabick JJ, Staveley-O'Carroll K, Billingsley EM, Marks JG, Helm KF: Eruptive latent metastatic melanomas after initiation of antitumor necrosis factor therapies. J Am Acad Dermatol 2007;56:S65-S67.

5 Lazar-Molnar E, Hegyesi H, Toth S, Falus A: Autocrine and paracrine regulation by cytokines and growth factors in melanoma. Cytokine 2000;12:547-554.

-6 Stern RS, Nichols KT, Vakeva LH: Malignant melanoma in patients treated for psoriasis with methoxsalen (psoralen) and ultraviolet A radiation (PUVA). The PUVA Follow-up Study. N Engl J Med 1997;336:10411045.

7 Buchbinder R, Barber M, Heuzenroeder L, Wluka AE, Giles G, Hall S, Harkness A, Lewis D, Littlejohn G, Miller $\mathrm{MH}$, Ryan PF, Jolley D: Incidence of melanoma and other malignancies among rheumatoid arthritis patients treated with methotrexate. Arthritis Rheum 2008;59:794-799.

8 Diwan AH, Lazar AJ: Nevoid melanoma. Clin Lab Med 2011;31:243-253.

McKee PH: Clues to the diagnosis of atypical melanocytic lesions. Histopathology 2010;56:100-111.

$\rightarrow 10$ Rongioletti F, Smoller BR: Unusual histological variants of cutaneous malignant melanoma with some clinical and possible prognostic correlations. J Cutan Pathol 2005;32:589-603.

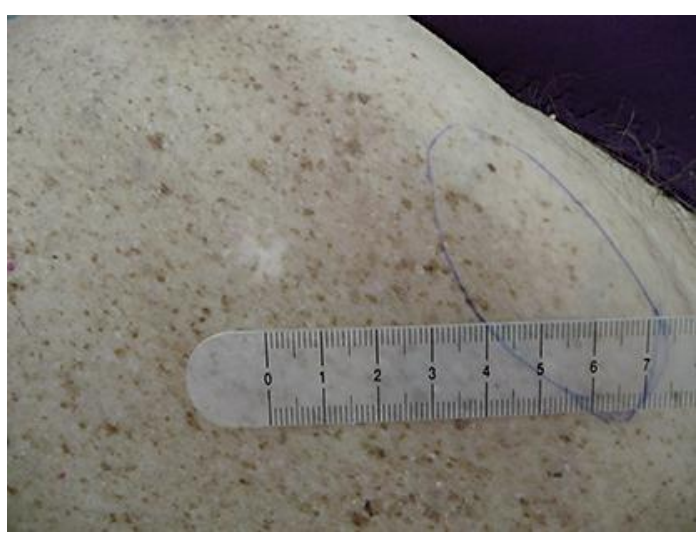

Fig. 1. The scar from a previously excised nevus located $4 \mathrm{~cm}$ below the metastatic inguinal lymphadenopathy. 
Safa et al.: Nodal Melanoma Metastasis under Infliximab Therapy in a Patient with Nevoid Melanoma First Misdiagnosed as Benign Nevus: A Potentially Dangerous Diagnostic Pitfall in the Era of Biologic Therapies
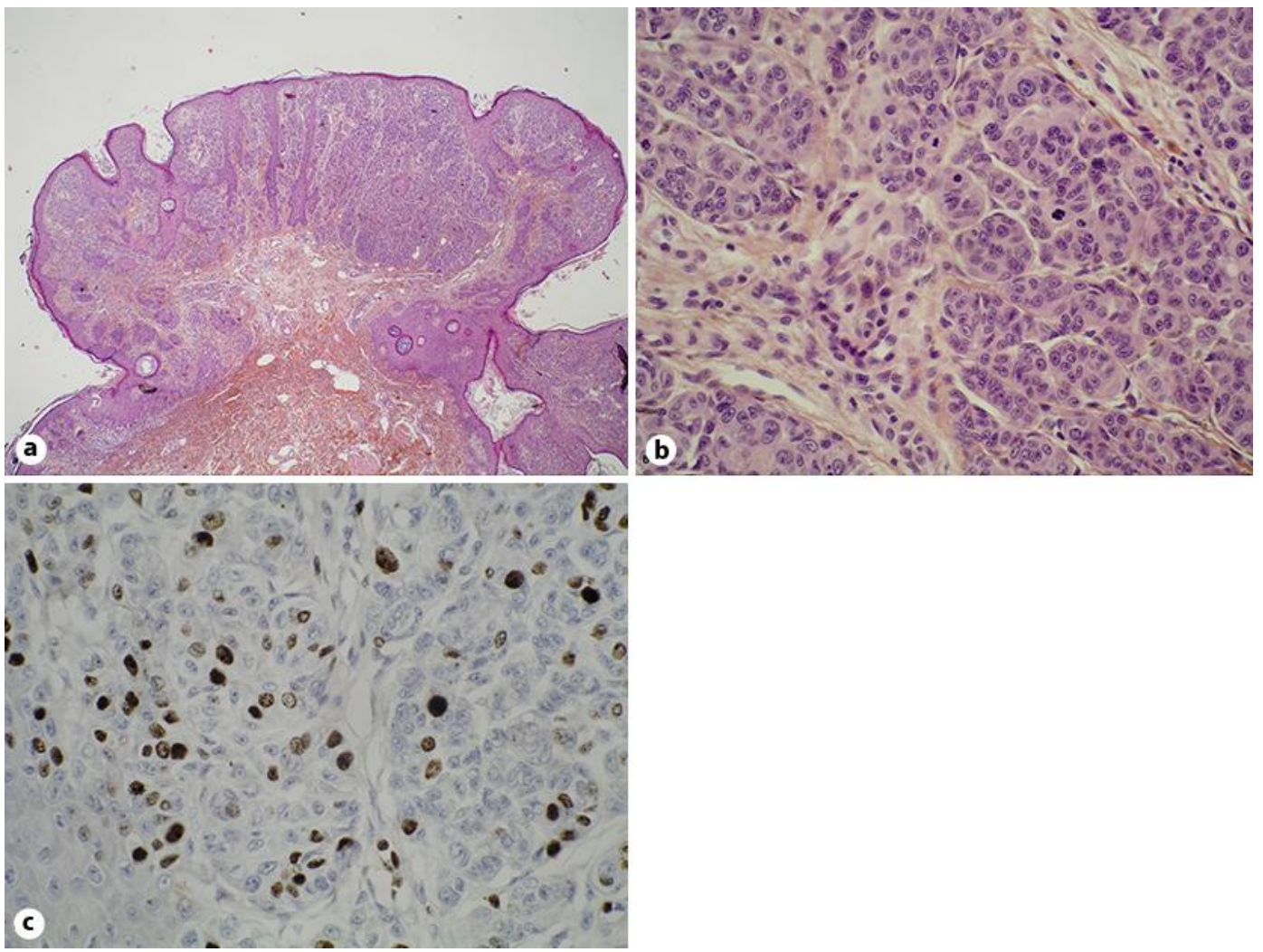

Fig. 2. a At low power, nevoid melanoma has the architecture of a banal compound nevus (HE staining, $\times 20$ ). Diagnostic clues included. $\mathbf{b}$ The presence of dermal mitotic figures ( 5 mitoses $/ \mathrm{mm}^{2}$ ) at high power (HE staining, $\times 80$ ). c A high Ki-67 immunoreactivity (Ki-67 index 20\%) in the lower parts of the lesion $(\times 80)$. 